K.A. van der Hucht, G. Koenigsberger \& P.R.J. Eenens, eds.

\title{
MERLIN observations of WR 147 (WN8(h)+B0.5V)
}

\author{
Sarah K. Watson ${ }^{1}$, Richard J. Davis ${ }^{1}$, and Peredur M. Williams ${ }^{2}$ \\ ${ }^{1}$ NRAL, Jodrell Bank, University of Manchester, UK \\ ${ }^{2}$ Royal Observatory, Blackford Hill, Edinburgh, UK
}

\section{Introduction}

WR 147 was first discovered to be a strong radio, X-ray and infra-red emission source by Caillault et al. (1985). It was classified as a WN8 type star with a very large mass loss rate and unusual radio emission $(\alpha<0)$ for a thermal source. This unusual behaviour was explained by MERLIN (Moran et al. 1989) and VLA (Churchwell et al. 1992) observations in which it was resolved into two components: a 'thermal' source associated with the Wolf-Rayet star and a non-thermal component $\sim 600$ mas to the north. Churchwell et al. showed that the southern component had the expected spectral index of $\alpha \simeq 0.6$ for free-free emission from a stellar wind. They also showed that the northern component had a spectral index of $\alpha \simeq-0.5$ and was therefore a non-thermal source, now thought to be due to a colliding wind region.

It is also evident that the radio emission from both sources varies - in structure and total flux - and we expect to get MERLIN observations of the system at three epochs in Semester 98B to examine this. The origin of the variations in the southern (WR wind) source - which may in turn cause variation in the non-thermal source after a delay $(\sim 1.7 \mathrm{yr})$ related to the wind travel time to the interaction region - is unknown.

The discovery in a UKIRT Service observation of a faint stellar companion to the WR star at the same position angle as the non-thermal radio source but a little more distant demonstrated that the non-thermal emission arose from the collision of the WR and companion's winds (Williams et al. 1997) - the first direct confirmation of a process previously inferred indirectly. The B0.5V companion star was found to be luminous enough to have a stellar wind of its own and the geometry of the non-thermal source (Figure 1) was shown to be consistent with emission from the collision of the WR and companion winds. The IR companion has since been observed in an optical image from the HST (Niemela et al. 1998), thereby confirming the colliding-wind geometry.

\section{Observations}

Two $5 \mathrm{GHz}$ MERLIN images of the Wolf-Rayet system WR 147 taken three years apart are shown here. Figure 1 shows the images from 1992 June 24 and 1995 April 29. No evidence of the bridge between the two component as seen by Churchwell et al. was detected in these images.

\subsection{The southern component WR 147S}

The peak intensity (and therefore the temperature) does not change between the two maps, but the total flux density detected, and hence the mass loss 


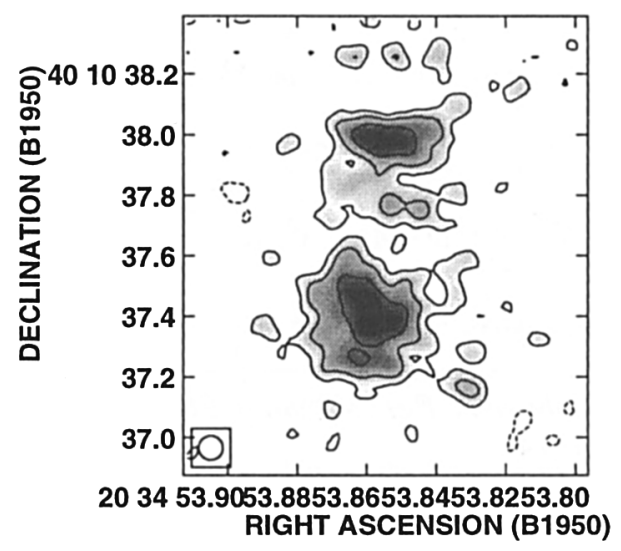

1992 June 24

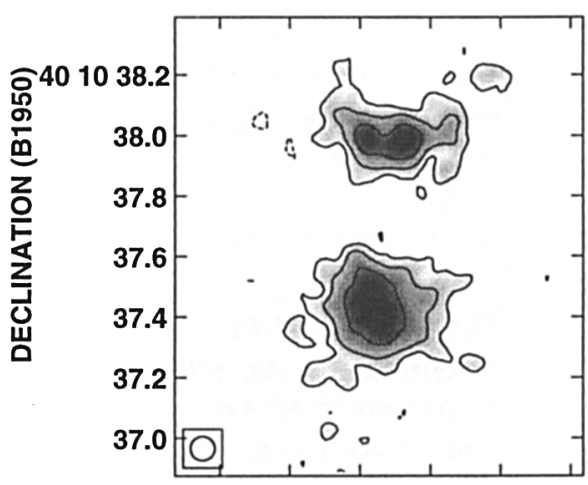

2034 53.9053.8853.8653.8453.8253.80 RIGHT ASCENSION (B1950)

1995 April 29

rate, and the size of the source decrease. This is the behaviour expected from a thermal source, a constant central temperature with size variations directly related to the mass loss rate variations of the WR star. The source is distinctly not spherically symmetric, as is usually assumed in models of stellar winds. There is also structure apparent within the source which changes between the observation dates. These factors will cause the mass loss rates calculated to be overestimates by as much as a factor of three.

\subsection{The northern component WR $147 \mathrm{~N}$}

The peak intensity decreases, while the total flux density detected increases. This variability implies that the production of non-thermal radiation in the region has decreased and some of the high energy material may be radiating away in a thermal manner, thereby increasing the size of the region. The structure within the source changes between observations. This may be due to the unsymmetrical behaviour of the WR's stellar wind, or it may be due to a decrease in momentum of the stellar wind. A decrease in momentum of the stellar wind would also explain the reduction in non-thermal radiation, but there is a timelag for the stellar wind to travel between the northern and southern components of $\sim 1.7$ years, so the reduction in mass loss rate seen in these two maps is not the cause of the reduction in non-thermal radiation seen at the same time.

\section{References}

Caillault, J.-P., Chanan, G.A., Helfand, D.J., et al. 1985, Nature 313, 376

Churchwell, E.B, Bieging, J.H., van der Hucht, K.A., Williams, P.M., et al. 1992, ApJ 393, 329

Moran, J.P., Davis, R.J., Spencer, R.E., Bode, M.F., et al. 1989, Nature 340, 449

Niemela, V.S, Shara, M.M., Wallace, D.J., Zurek, D.R., et al. 1998, AJ 115, 2047

Williams, P.M., Dougherty, S.M., Davis, R.J., van der Hucht, K.A., et al. 1997, MNRAS 289, 10 